\title{
DESCRIPCIÓN DE LA LARVA DE PARACLES AZOLLAE (BERG, 1877) (LEPIDOPTERA: EREBIIDAE)
}

\author{
Enrique Morelli; Andrea Diez; Gabriela Bentancur-Viglione*
}

\author{
Sección Entomología, Facultad de Ciencias, UdelaR, Montevideo, Uruguay \\ ${ }^{\star}$ Autor para correspondencia: gbentancur@fcien.edu.uy
}

\section{RESUMEN}

La descripción de estados inmaduros para especies de lepidópteros acuáticos es fragmentaria y casi inexistente para el continente Americano. Las larvas de las especies acuáticas de Arctiinae se reconocen por la presencia de sedas secundarias y verrugas, otorgándoles un aspecto piloso. El género Paracles está presente en la región Neotropical, donde Uruguay y Argentina son considerados el límite sur de su distribución. Para Uruguay están citadas las especies Paracles azollae (Berg, 1877), P. tenuis (Berg, 1877), P. burmeisteri (Berg, 1877), P. uruguayensis (Berg, 1886), P. cajetani (Rothschild, 1910) y P. vulpina (Hübner, [1825]). La larva madura de $P$. azollae se caracteriza por un escudo protoráxico con sedas principales y verrugas. T2 y T3 con un complejo plastrón respiratorio dorsal y verrugas de sedas plumosas, largas y gruesas. A1-2 con plastrón dorsal complejo con sedas en calazas. A3-8 con plastrón y sedas sobre calazas. A9 con plastrón complejo y verrugas. A10 con verruga dorsal. La presencia y el número de verrugas, el número y la forma de las sedas verrucales y la ubicación del plastrón aerífero son presentados como caracteres anatómicos para la determinación específica del género Paracles.

Palabras clave: Entomología acuática; inmaduros; taxonomía larval; Arctiinae.

\section{ABSTRACT}

Description of the larva of Paracles azollae (Berg, 1877) (Lepidoptera: Erebiidae). The description of immature stages for aquatic lepidopteran species is fragmentary and almost non-existent for the American continent. The larvae of the aquatic species of Arctiinae are recognized by the presence of secondary setae and warts, which give them a hairy appearance. The Paracles genus is present in the Neotropical regions, where Uruguay and Argentina are considered the southern limit of their distribution. For Uruguay are cited the species Paracles azollae (Berg, 1877), P. tenuis Berg, 1877, P. burmeisteri (Berg, 1877), P. uruguayensis (Berg, 1886), P. cajetani (Rothschild, 1910) and $P$. vulpina (Hübner, [1825]). The mature larva of $P$. azollae is characterized by a protoraxic shield with main setae and warts. T2 and T3 with dorsal respiratory plastron complex, and warts with long and thick feathery setae. A1-2 with a complex dorsal plastron with setae in pinaculum. A3-8 with plastron and setae in pinaculum. A9 with complex plastron and warts. A10 with dorsal wart. The presence and number of warts, the number and shape of setae and the location of the plastron are presented as anatomical characters for the specific determination of the Paracles genus.

Key words: Aquatic entomology; immature; larval taxonomy; Arctiinae

\section{INTRODUCCIÓN}

De las 11.000 especies descriptas para la subfamilia Arctiinae (Erebiidae), 6000 están distribuidas en el Neotrópico (Watson \& Goodger, 1986; Heppner, 1991; Domínguez \& Fernández, 2009; Meneses et al., 2013). Los insectos adultos son de tamaño mediano a grande, de colores brillantes, aposemáticos o miméticos (Domínguez \& Fernández, 2009). Las larvas se caracterizan por la presencia de numerosas sedas secundarias organizadas sobre verrugas que les dan una apariencia muy pilosa. Aceptan una amplia variedad de plantas huésped desde algas, gramíneas o arbustos y árboles deciduos (Lange, 1984). Los Arctiinae incluyen especies con larvas de vida subacuática, las cuales además de los caracteres comunes al resto se caracterizan por presentar penachos de sedas de colores oscuros, rojizos, blanquecinos. Hasta el momento, la descripción de los caracteres de la anatomía externa larval de los Arctiinae acuáticos es muy fragmentaria y no son considerados dentro de las claves del género (Drechsel, 2014). Romero \& Navarro (2009), en su clave para larvas de las familias acuáticas y semiacuáticas de lepidópteros, sólo toman como caracteres distintivos del género la distancia entre las metacoxas y la presencia de verrugas prominentes, sin especificar su ubicación y número.

El género Paracles (Walker, 1855) es exclusivamente neotropical, con 76 especies descriptas (Berg, 1875a, 1875b, 1877a, 1877b, 1877c, 1877d). Las larvas de este género poseen hábitos polífagos no específicos, ha sido asociado al camalote Eichhornia crassipes Solms, 1883.y a otras plantas acuáticas relacionadas (Domínguez \& Fernández, 2009). Para Uruguay están citadas: Paracles azollae (Berg, 1877), Paracles burmeisteri (Berg, 1877), P. tenuis (Berg, 1877), P. cajetani (Rothschild, 1910), P. uruguayensis (Berg, 1886) y P. vulpina (Hübner, [1825]) (Berg, 1877b, 1877c; Beccacece et al., 2014; Biezanko et al. 1957, 1978; Vincent \& Laguerre, 2014).

Paracles azollae fue descripta originalmente por Berg, 1877 en el género Palustra quien publicó la 
descripción de la larva y su biología mencionando solamente caracteres de coloración y planta hospedera. En este trabajo se describe la larva de $P$. azollae y se ilustran los principales caracteres anatómicos de valor taxonómico para la identificación de la especie.

\section{MATERIAL Y MÉTODOS}

Se recolectaron seis larvas maduras sobre vegetación emergente en un cuerpo de agua en la localidad de Sierra de Ánimas en el departamento de Maldonado, Uruguay, en agosto de 2014. Se fijaron dos larvas en alcohol $70^{\circ}$. Las restantes fueron criadas, hasta la emergencia del imago. Para la determinación se utilizaron las claves de Romero y Navarro (2009) y Carter y Kristensen (1998) y la confirmación específica del adulto la realizó el Dr. Hernán Beccacece (Universidad de Córdoba, Argentina).

Los esquemas para las descripciones anatómicas se realizaron en cámara clara con microscopio estereoscópico, utilizando la terminología larval propuesta por Hinton (1946), con las modificaciones de Sterhr (1987). Se empleó la denominación CD para el grupo de sedas cefalodorsal siguiendo a Duarte et al. (2005). La anchura de la cápsula cefálica se midió en las larvas y en la exuvia larval. Tanto el ejemplar adulto hembra y las larvas fueron depositados en la colección entomológica de Facultad de Ciencias (FCELP).

\section{RESULTADOS}

Redescripción de la larva madura de Paracles azollae.

Larva eruciforme, hipognata, de longitud máxima $32,0 \mathrm{~mm}$. Aspecto pubescente, erizado de sedas punzantes (Fig. 1). Coloración general negro azabache,

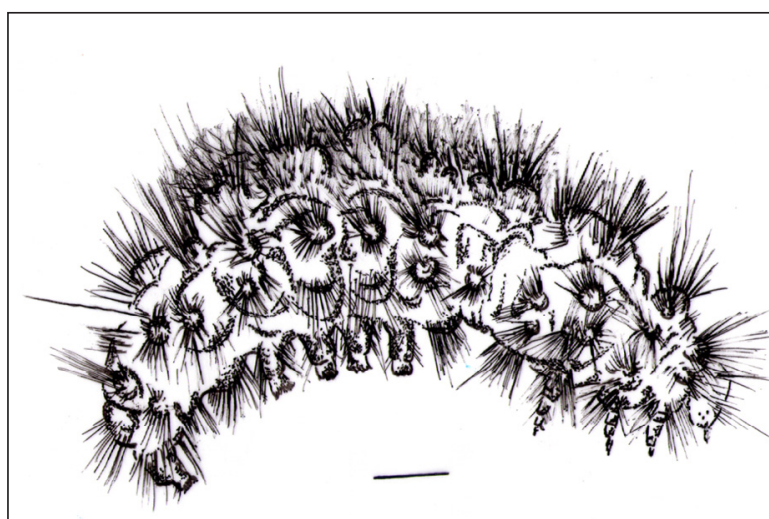

Fig. 1. Vista lateral de la larva madura de $P$. azollae. Escala: $4 \mathrm{~mm}$. verrugas laterales rojizas y plastrón corto dorsal blanco. Cabeza cuadrangular de contorno redondeado. Ancho máximo $3,0 \mathrm{~mm}$ y longitud desde el borde del clípeo 2,5 mm. Tegumento cefálico liso, castaño oscuro brillante con poros claros, zona adfrontal más oscura. Piezas bucales castaño oscuro, áreas incisivas de la mandíbula más oscura y con tres dientes incisivos bien diferenciados. Base de las antenas blanquecina. Sedas en general, castaño claro. Frente triangular, cónica de $1 \mathrm{~mm}$. de ancho máximo. Espiráculos presentes en $\mathrm{T}_{1}$ y $\mathrm{A}_{1-8}$.

\section{Quetotaxia cefálica (Fig. 2. a y b)}

Tres sedas propioceptoras $\left(\mathrm{MD}_{1,2,3}\right)$; dos poros verticales $(\mathrm{Pa}, \mathrm{Pb})$; dos sedas anteriores $(\mathrm{A} 1, \mathrm{~A} 2$,$) ; dos$ sedas estematales (S1,S2); dos sedas adfrontales (AF1, AF2); un poro adfrontal (AFa) a cada lado; dos sedas clipeales $(\mathrm{C} 1, \mathrm{C} 2)$; seis estematas, cuatro en semicírculo, 5 y 6 hacia la base genal con $\mathrm{S} 1$ en el centro. Antenas triarticuladas, primer artículo muy ensanchado, segundo segmento alargado, dos veces el largo del primero primer artículo ensanchado,

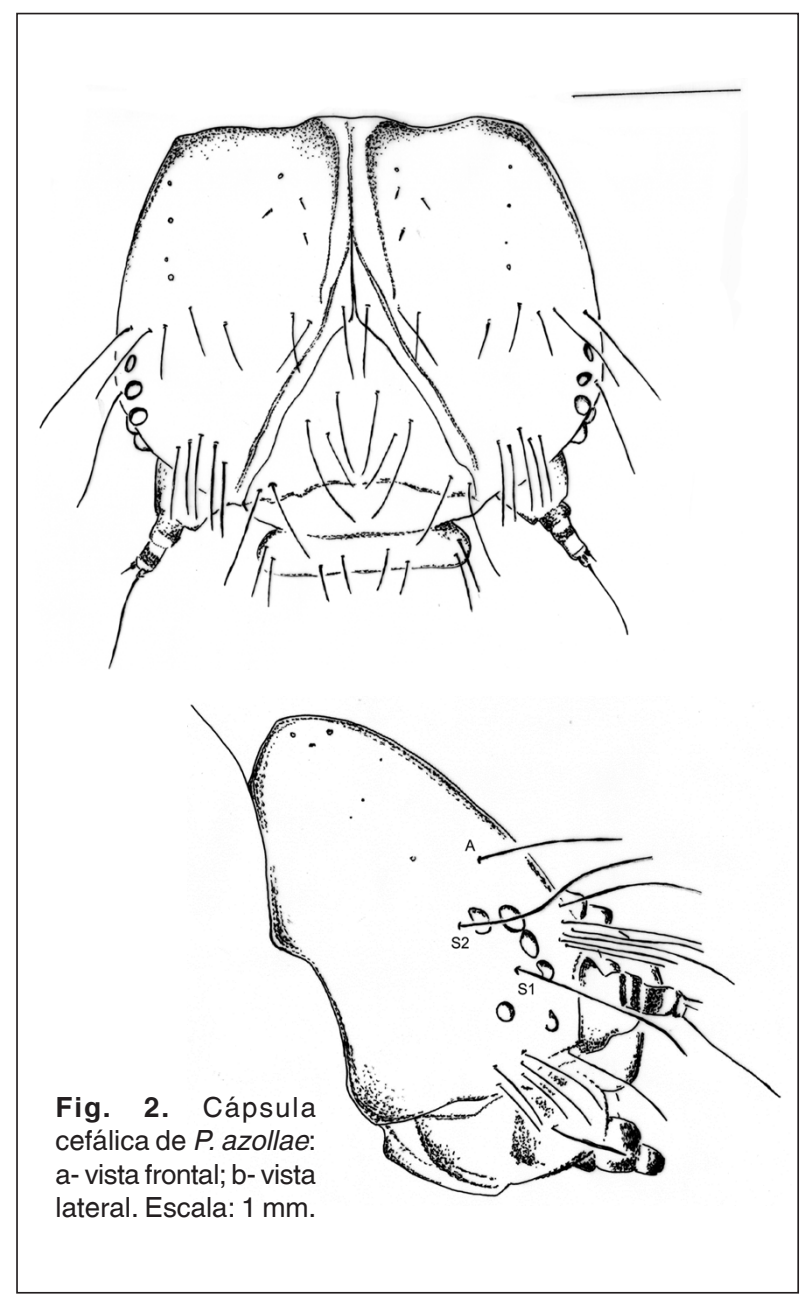


segundo más alargado y tercer segmento muy pequeño con una larga seda terminal.

\section{Tórax}

Escudo protorácico (Ep) (Fig. 3a) con dos áreas triangulares de ápice romo, 16-18 sedas plumosas largas y rígidas, en calaza, castaño oscuras, intercaladas con 14-16 sedas plumosas medianas y finas. El conjunto de sedas está más agrupado en forma de franja sobre el borde occipital. Una verruga subdorsal (Vsd) y supraespiracular a cada lado, formada por una seda central plumosa rígida, castaño oscura, enmarcada por 4-6 sedas más cortas. Una verruga lateral $(\mathrm{VI})$ a cada lado, formada por 10-12 sedas plumosas rígidas, castaño oscuras, sobre calaza intercaladas por 6-8 sedas similares más finas y claras. Una verruga subventral (Vsv) a cada lado, formada por 10 sedas plumosas rígidas, castaño oscuras sobre calazas, rodeada por 12-14 sedas más finas y claras.

T2 y T3 (Fig 3b). Plastrón dorsal respiratorio (PI) formado por dos penachos enfrentados de sedas plumosas blanquecinas muy apretadas. Una verruga dorsal (Vd) a cada lado, formada por 14-16 sedas plumosas largas, gruesas, castaño oscuras, sobre calaza. En el margen de la verruga se insertan 4-6 sedas similares castaño claras, 6-8 sedas cortas y rígidas sobre calaza pequeña, intercaladas. Una verruga subdorsal (Vsd) a cada lado, con 14-16 sedas plumosas, alargadas, gruesas, castaño oscuras sobre calaza. 2-4 sedas similares castaño claras sobre calaza, intercaladas con 6-8 sedas cortas castaño oscuras sobre calaza. Una verruga lateral (VI) de cada lado formado por 12-14 sedas plumosas alargadas, gruesas, castaño oscuras sobre calaza. 14-16 sedas plumosas castaño claras sobre calaza, ubicadas en el contorno de la verruga intercaladas con dos sedas cortas oscuras sobre calaza pequeña. Una verruga subventral (Vsv) a cada lado formada por ocho sedas plumosas largas

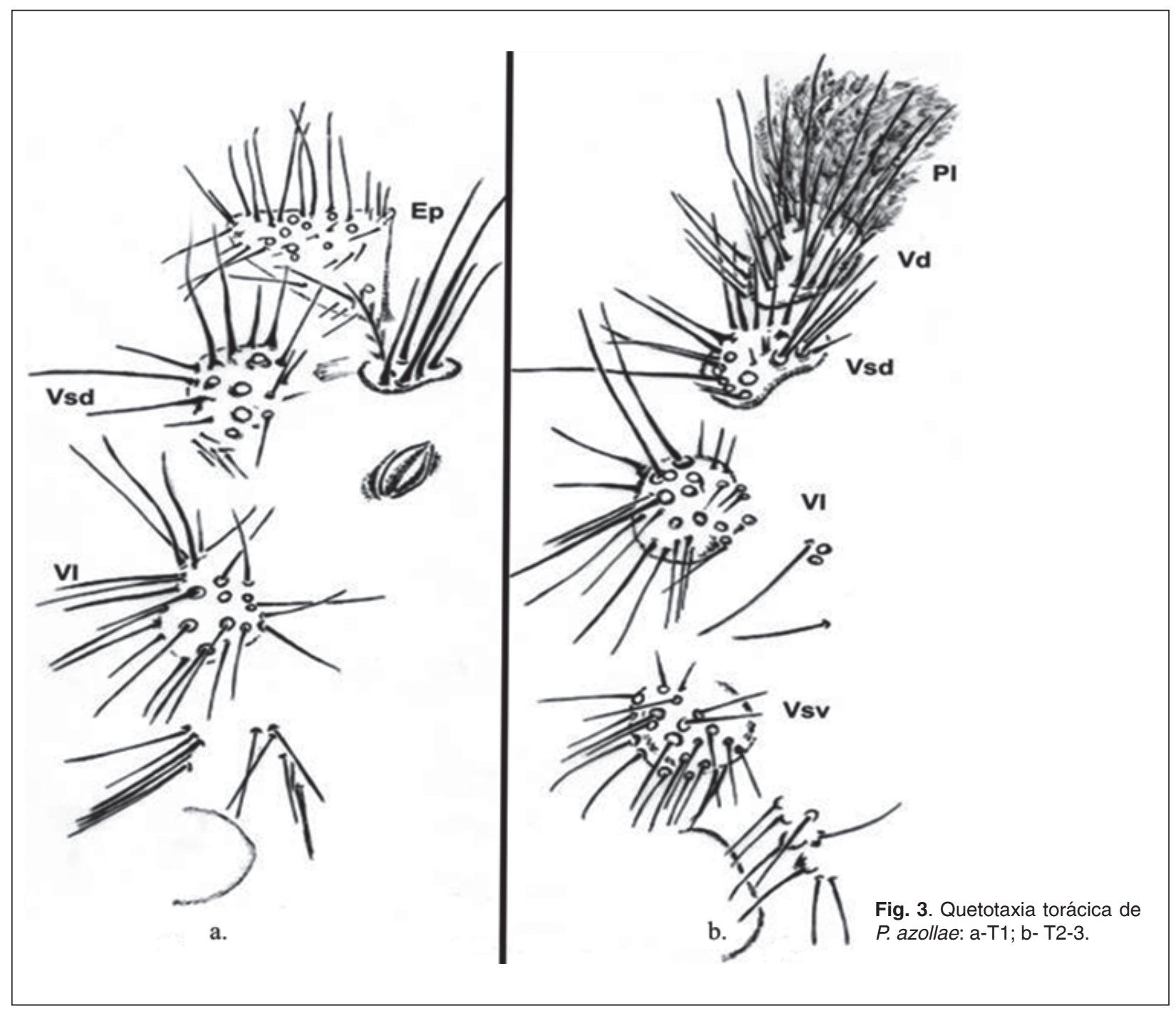

Bol. Soc. Zool. Uruguay (2ª época). 2018. Vol. 27 (2): 34-40 ISSN: 0255-4402 

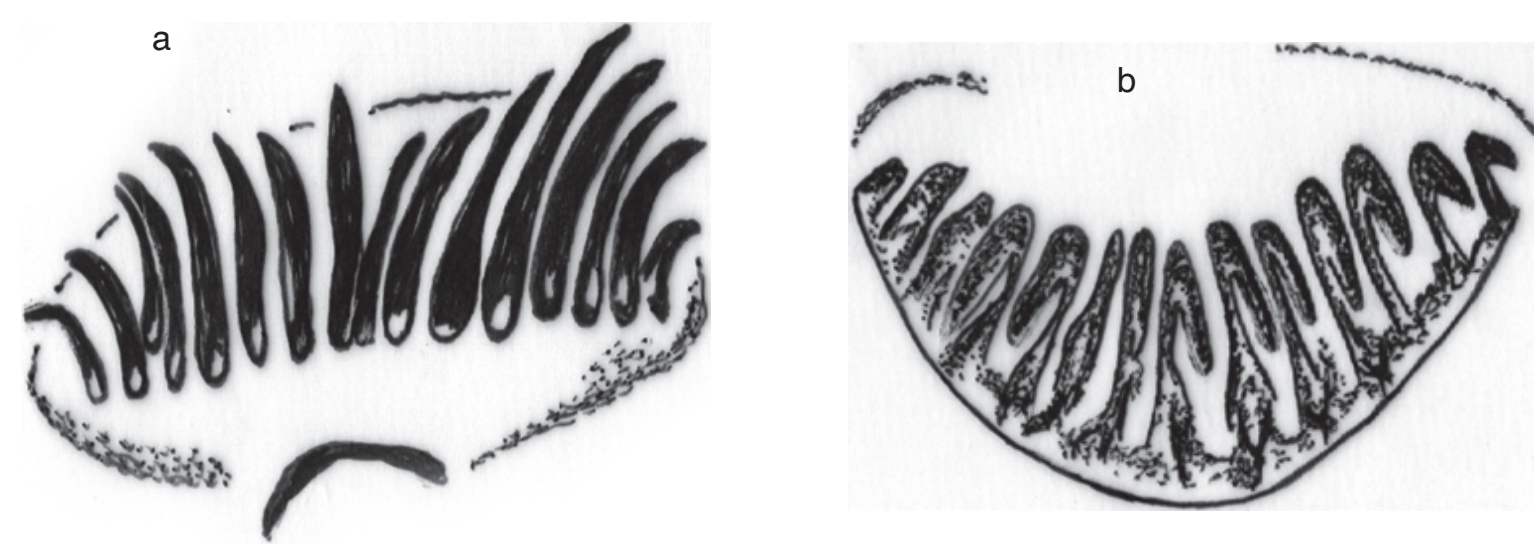

Fig. 4. Esquema del diseño espurripedial de $P$. azollae: a. segmentos A3-6; segmento A10.

gruesas castaño oscuras, sobre calaza. 16-18 sedas plumosas, alargadas, castaño claras sobre calaza, ubicado principalmente sobre el borde de la verruga; dos sedas cortas centrales sobre calaza pequeña.

\section{Abdomen}

Espurrìpedios presentes en los segmentos $A_{3-6}$ y $A_{10}$. En $A_{3-6}$ crochets homoideos uniseriales, $A_{3-}$ ${ }_{6}$ con 16 crochets y $A_{10}$ con 14 crochets (Fig. 4 a y b).

A 1-2. (Fig 5.) Plastrón dorsal formado por cuatro cepillos entrelazados (dos a cada lado) de sedas plumosas y finas, ambarinas. Estos cepillos de sedas son compactos y se entrecruzan sobre el espacio dorsal de la larva, originándose en los bordes internos de las verrugas dorsales. Otros tres cepillos de sedas plumosas rígidas, cortas, castaño oscuras, y muy apretados se originan en los bordes externos de las verrugas dorsales y en el borde interno de la subdorsal.

$\mathrm{Vd}$, dos a cada lado, 1-2 similares formadas por 14-16 sedas plumosas, rígidas, alargadas, castaño ambarinas sobre calazas oscuras, bastante regular en su distribución; pocas sedas medianas finas centrales sobre calaza pequeña. En el área media del cuerpo se distingue una zona de poros pequeños de color oscuro.

Una $\mathrm{Vl}_{1}$ a cada lado, arriñonada en vista lateral, formada por 20-22 sedas largas, plumosas, rígidas, oscuras en el primer tercio y ambarinas hacia el ápice, todas con calazas gruesas y oscuras. 10-12 sedas plumosas, medianas, oscuras, intercaladas.

Una $\mathrm{VI}_{2}$ a cada lado, de borde redondeado, más pequeña que $\mathrm{VI}_{1}$, con 20-22 sedas alargadas, rígidas, sobre calaza oscura, de distribución regular y más uniformes en su coloración ambarina. 2-4 sedas medianas externas, sobre calazas pequeñas.

Una Vsv a cada lado, pequeña y alargada con 20 sedas rígidas formando tres hileras claramente visibles, la ventral con 4 cuatro sedas sobre calazas gruesas y oscuras, las laterales con ocho sedas ambarinas sobre calaza oscura.

Una $\mathrm{Vv}_{1}$ a cada lado, alargada transversalmente con 20-22 sedas alargadas, ambarinas, sobre calaza oscura, de ubicación regular en toda la superficie. Se destaca una seda central gruesa en calaza.

Una $\mathrm{Vv}_{2}$ a cada lado, pequeña, de contorno circular, con 12-14 sedas finas castaño ambarinas, más alargadas hacia el borde distal.

A3-6 $\mathrm{Vd}_{1} \mathrm{yVd}_{2}$, una verruga subdorsal, y una $\mathrm{V}_{1}$. Dos $\mathrm{SV}_{1,2}$ a cada lado de forma similar, alargadas lateralmente con $28-30$ sedas plumosas y rígidas. En el área proximal se ubican las sedas medianas, en el área distal se localizan sedas más largas, rígidas y plumosas, sobre calazas oscuras. No hay sedas cortas. Las sedas se ubican en 10 líneas transversales y regulares de 3-4 unidades cada una.

$A_{7}$ Verrugas dorsales, subdorsales igual que $A_{1-2}$; verrugas lateral y subventrales como en $A_{3-6}$.

Verrugas ventrales $V v_{1}$ y $V v_{2}$ similares en su forma, $\mathrm{V}_{2}$ de mayor tamaño, con $12-14$ sedas rígidas castaño más oscuras en la base sobre calazas, siempre la más pequeña en borde proximal y tres hileras de sedas más largas a partir de la mitad distal de la verruga.

$\mathbf{A}_{8}$ similar a $\mathbf{A}_{7}$

$\mathbf{A}_{9}$ con un plastrón con dos cepillos de sedas claras entrecruzadas y sedas plumosas que se originan en el borde interno de la Vd. Una gran Vd a cada lado castañas, con 14-16 sedas gruesas bandeadas, oscuras, prominente, ubicada a lo largo del área central. Sedas 


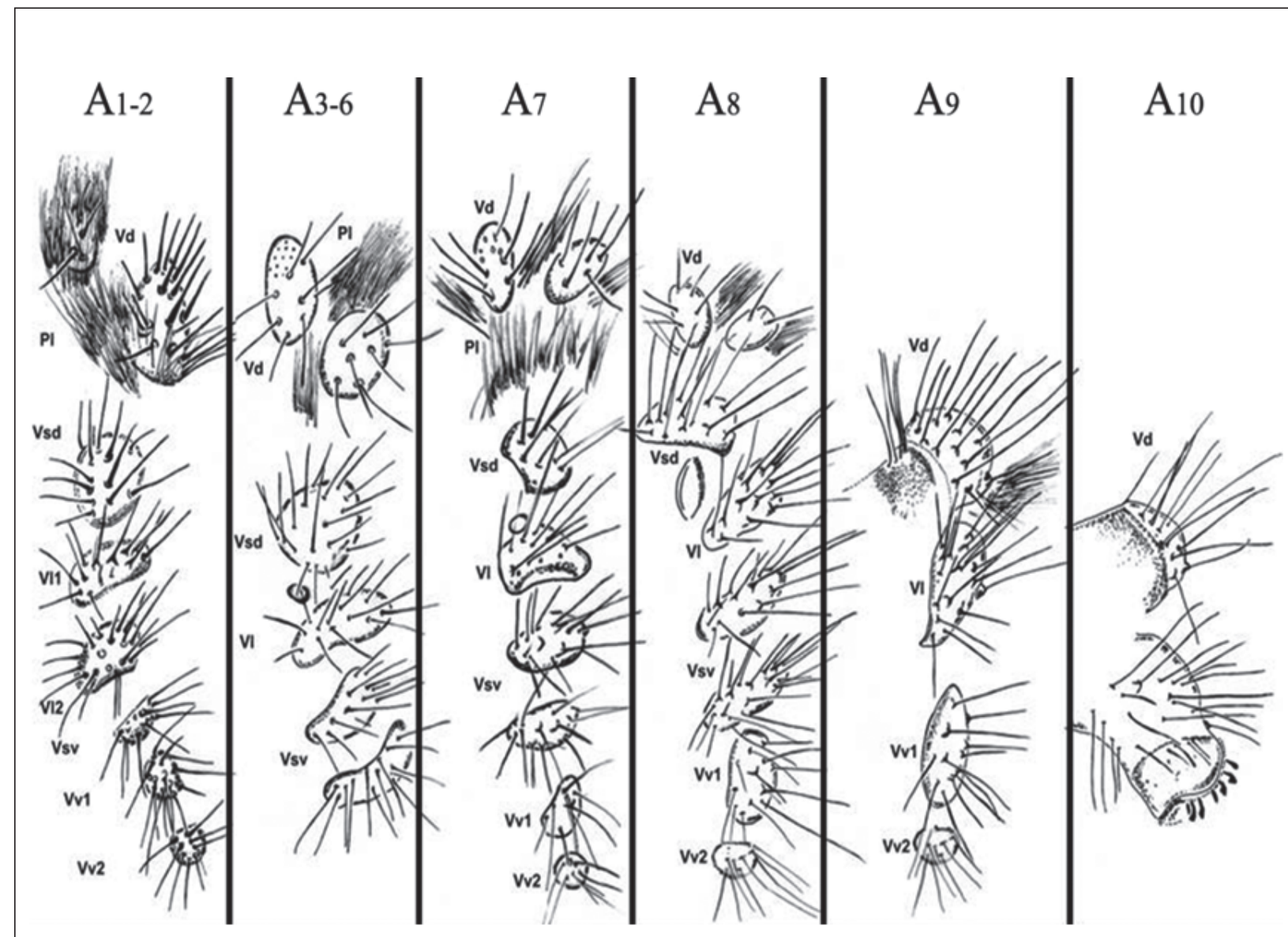

Fig. 5. Quetotaxía del abdomen en vista lateral de P. azollae.

medianas más finas y oscuras en el borde de la verruga. Una VI de borde arriñonado con 20-22 sedas rígida de calaza oscura, las sedas centrales más largas y algunas sedas más oscuras y pequeñas distribuidas en el centro. $\mathrm{Vv}_{1}$ a cada lado con 8 sedas largas en calaza oscura y cuatro sedas medianas sobre calaza pequeña. $\mathrm{Vv}_{2}$ pequeña, discreta, con gran seda alargada, en calaza oscura, con siete sedas medianas rígidas.

$\mathbf{A}_{10}$ con una verruga dorsal de cada lado que parecen unirse en la zona media, con sedas rígidas, plumosas, y base oscura intercaladas con sedas pequeñas y medianas también oscuras. Alrededor de 20 sedas secundarias cortas, rígidas, plumosas, distribuidas de forma regular ocupando todo el espurrípedio.

\section{DISCUSIÓN}

Las descripciones de las larvas de Paracles burmeisteri, P. argentina, P. laboulbeni y P. tenuis sólo contemplan la coloración general del cuerpo y mencionan la presencia de verrugas sedosas (Berg, 1877a, 1877c; Bar, 1873). La descripción larval original de $P$. azollae (= Palustra azollae) realizada por Berg, (1877a) sólo toma en cuenta el color general del cuerpo y de las manchas torácicas. Las recientes descripciones de Paracles palustris (Joergensen, 1935), Paracles fusca (Walker, 1856), Paracles contraria Walker, 1856 y Paracles aurantiaca (Rothschild, 1910) realizadas por Drechsel y Drechsel (2014, 2016a, 2016b, 2017) describen la coloración del complejo de sedas y verrugas de los distintos estados larvales. El presente trabajo aporta como información complementaria a las claves de larvas de lepidópteros semiacuáticos (Romero \& Navarro, 2009; Carter y Kristensen, 1998), una descripción detallada de la quetotaxia larval para cada uno de los segmentos del cuerpo, distinguiendo la presencia de los distintos tipos y grupos de sedas, la ubicación de las verrugas sedosas y la localización de los plastrones dorsales. Un cuadro comparativo del número y ubicación de las de verrugas torácicas y abdominales es presentado en la Tabla I. 
Tabla. I. Cuadro comparativo del patrón verrugal en los segmentos torácicos y abdominales de $P$. azollae.

\begin{tabular}{llllllllll} 
Segmentos & T1 & T2-3 & A1-2 & A3-6 & A7 & A8 & A9 & A10 \\
\hline Plastrón dorsal & 0 & + & + & + & + & + & + & 0 \\
Verruga dorsal1 & 0 & + & + & + & + & + & + & + \\
Verruga dorsal2 & 0 & 0 & + & + & + & + & 0 & 0 \\
Verruga subdorsal1 & + & + & + & + & + & + & 0 & 0 \\
Verruga lateral1 & + & + & + & + & + & + & + & 0 \\
Verruga lateral2 & 0 & 0 & + & 0 & 0 & 0 & 0 & 0 \\
Verruga subventral1 & + & + & + & + & + & + & 0 & 0 \\
Verruga subventral2 & 0 & 0 & 0 & 0 & + & + & 0 & 0 \\
Verruga ventral1 & 0 & 0 & + & 0 & + & + & + & 0 \\
Verruga ventra2 & 0 & 0 & + & 0 & + & + & + & 0 \\
\hline
\end{tabular}

\section{AGRADECIMIENTOS}

Agradecemos a los revisores que con sus comentarios mejoraron sustancialmente este trabajo.

\section{BIBLIOGRAFÍA}

Bar C. 1873. Sur un genre nouveau de Lépidoptères de la tribu des Bombycidae et dont la chenille est aquatique. Annales de la Société Entomologique de France, 3(5): 297-306.

Beccacece H., Vincent B. \& Navarro F. 2014. The type-material of Arctiinae (Lepidoptera, Erebidae) described by Burmeister and Berg in the collection of the Museo Argentino de Ciencias Naturales Bernardino Rivadavia (Buenos Aires, Argentina). ZooKeys, 421: 6589. doi: 10.3897/zookeys.421.6666.

Berg C. 1875a. Lepidópteros patagónicos observados en el viaje de 1874. Acta Académica Nacional. Cordoba, 1(4):63-102, (7): 155-158.

Berg C. 1875b. Patagonische Lepidopteren beobachtet auf einer Reise im Jahre 1874. Bulletin de la Société Impériale des Naturalistes de Moscou, 49(4): 191-247.

Berg C. 1877a. In: Dohrn CA. Drei Leseblumen. Stettiner Entomologische Zeitung, 38: 256-260.

Berg C. 1877b. Observations Lepidopterologiques II Descriptions de deux nouveaux Lépidoptéres de la famille des Arctiadae (Palustra azollae et Palustra tenuis). Annales de la Socété Entomologique de France, 7(5): 189-194.

Berg C. 1877c. Estudios Lepidopterológicus acerce de la fauna Argentina y Oriental. Anales de la Sociedad Científica Argentina, 3: 228-242, pl.

Berg C. 1877d. Observations Lepidopterologiques I. Remarques sur une Nouvelle chenille aquatique.
Annales de la Socété Entomologique de France, 7(5): 183-188.

Biezanko C. M., Ruffinelli A. \& Carbonel C.S. 1957. Lepidoptera del Uruguay. Lista anotada de especies. Revista de la Facultad de Agronomía, Montevideo, 46: 1-152.

Biezanko C. M., Ruffinelli A. \& Link D. 1978. Catálogo de Lepidópteros do Uruguai.- Revista Do Centro de Ciencias Rurais (suplemento):1-84.

Carter D. J. \& Kristensen N.P. 1998. Classification and Keys to higher taxa. En: Kristensen N. P. (Ed). Lepidoptera, Moths and Butterflies, Volumen 1: Evolution, Systematic, and biogeography, pp. 27-40. Handbuch der Zoologie Band IV Arthropoda: Insecta. Teilband/part 35. Walter de Gruyter. Berlin. New York.

Dominguez E. \& Fernandez H.R. 2009. Macroinvertebrados bentónicos sudamericanos: sistemática y biología. 1a ed. Tucumán: Fundación Miguel Lillo. 656 pp.

Drechsel U. 2014. Aquatic habit of larval instars of Paracles palustris (Joergensen, 1935) (Lepidoptera: Erebidae: Arctiinae). Paraguay Biodiversidad 1(18): 89-94.

Drechsel U. \& Drechsel García, S. 2016a. The immature stages of Paracles contraria Walker, 1855 (Lepidoptera: Erebidae: Arctiinae). Paraguay Biodiversidad, 3(6): 33-38.

Drechsel U. \& Drechsel García S. 2016b. The early stages of Paracles fusca (Walker, 1856) (Lepidoptera: Erebidae: Arctiini). Paraguay Biodiversidad, 3(8): 47-52.

Drechsel U. \& Drechsel S. 2017. The early stages of Paracles aurantiaca (Rothschild, 1910) (Lepidoptera: Erebidae: Arctiini) Paraguay Biodiversidad, 4(1): 1-7.

Duarte M., Robbins R. K. \& Mielke O. H. 2005. Immature stages of Calycopis caulonia (Hewitson, 1877) (Lepidotera, Lycaenidae, 
Theclinae, Eumaeini), with notes on rearing detritivorous haisstreaks on artificial diet. Zootaxa, 1063:1-31.

Heppner J. B. 1991. Faunal regions and the diversity of Lepidoptera. Tropical Lepidoptera, 2, supplement, 1: 1-85.

Hinton H.E. 1946. On the homology and nomenclature of the setae of lepidopterous larvae, with some notes on the phylogeny of the lepidoptera.Transaction of the Royal Entomological Society of London, 97: 1-37.

Lange W.H. 1984. Aquatic and semiaquatic Lepidoptera. En: Merritt, R.W. \& Cummins K. W. (Eds.). An introduction to the aquatic insects of North America, pp 348-360. Second edition. Kendall/Hunt Publ. Co., Dubuque, IA.

Meneses A.R., Bevilaqua M.V.O., Hamada N. \& Querino R.B. 2013. The aquatic habit and host plantas of Paracles klagesi (Rothschild) (Lepidoptera, Erebiidae, Arctiinae) in Brazil. Revista Brasileira de Entomologia, 57(3): 350352.
Romero V.F. \& Navarro F. 2009. Lepidoptera. En: Domínguez y Fernández (Eds.) Macroinvertebrados Bentónicos sudamericanos. Sistemática y Biología, pp. 309-340. Fundación Miguel Lillo, Tucumán, Argentina.

Stehr F.W. 1987. Cap. 26. Order Lepidoptera. En: F. W: Stehr (Ed.) Immature insects, Volume 1, pp. 754. Kendall-Hunt, Dubuque, lowa. Quarto. Hard cover.

Vincent B. \& Laguerre M. 2014. Catalogue of the Neotropical Arctiini Leach, [1815] (except Ctenuchina Kirby, 1837 and Euchromiina Butler, 1876) (Insecta, Lepidoptera Erebidae, Arctiinae). Zoosystema 36 (2): 137-533. http://dx.doi.org/ $10.5252 / z 2014 n 2 a 1$

Watson A \& Goodger D.T. 1986. Catalogue of the Neotropical Tiger-Moths. Occasional Papers on Systematic Entomology, British Museum (Natural History), No $1.71 \mathrm{pp}$.

Fecha de Recibido: 6 de marzo de 2018 Fecha de Aceptado: 23 de agosto de 2018 